\title{
Alignment adjustment using the Valgus stress technique can increase the surgical accuracy of novice surgeons during medial opening-wedge high Tibial osteotomy
}

\author{
Man Soo Kim¹, In Jun Koh², Yong Gyu Sung ${ }^{1}$, Dong Chul Park', Sung Bin Han ${ }^{1}$ and Yong $\ln ^{1^{*}}$
}

\begin{abstract}
Background: The purpose of this study was to compare the degree of accuracy of coronal alignment correction with use of the "alignment adjustment under valgus stress technique" between expert and novice surgeons during medial opening-wedge high tibial osteotomy (MOWHTO).
\end{abstract}

Methods: Forty-eight patients who underwent MOWHTO performed by an expert surgeon (expert group) and 29 by a novice surgeon (novice group) were enrolled in analysis. During surgery, lower-extremity alignment was corrected using the "alignment adjustment under valgus stress technique". Normocorrection was defined as a weight-bearing line ratio between 55 and $70 \%$ and the correction accuracy was compared between expert and novice groups using the ratio of normocorrection to outliers. The clinical outcomes were also compared using the Western Ontario and McMaster Universities Osteoarthritis Index (WOMAC) at 1 year after surgery.

Results: The undercorrection rate was $14.6 \%$ in the expert group and $13.8 \%$ in the novice group, while the overcorrection rate was $2.1 \%$ in the expert group and $3.4 \%$ in the novice group. In the ratio of normocorrection to outliers, no difference was found between the two groups at the one-year follow-up visit (83.3\% in the expert group vs. $82.8 \%$ in the novice group; $p>0.05$ ). Also, no significant differences were seen in WOMAC subscores immediately preoperatively and at 1 year after surgery (all $p>0.05$ ).

Conclusion: Adhering to the "alignment adjustment under valgus stress technique" protocol enabled novice surgeons to achieve similar surgical accuracy as that of an expert surgeon in coronal alignment during MOWHTO.

Level of evidence: Level III.

Keywords: Novice, Expert, High Tibial osteotomy, Alignment, Correction, Accuracy, Weight-bearing line

\footnotetext{
*Correspondence: iy1000@catholic.ac.kr

'Department of Orthopaedic Surgery, Seoul St. Mary's Hospital, College of Medicine, The Catholic University of Korea, 222, Banpo-daero, Seocho-gu, Seoul 06591, Republic of Korea

Full list of author information is available at the end of the article
}

(c) The Author(s). 2021 Open Access This article is licensed under a Creative Commons Attribution 4.0 International License, which permits use, sharing, adaptation, distribution and reproduction in any medium or format, as long as you give appropriate credit to the original author(s) and the source, provide a link to the Creative Commons licence, and indicate if changes were made. The images or other third party material in this article are included in the article's Creative Commons licence, unless indicated otherwise in a credit line to the material. If material is not included in the article's Creative Commons licence and your intended use is not permitted by statutory regulation or exceeds the permitted use, you will need to obtain permission directly from the copyright holder. To view a copy of this licence, visit http://creativecommons.org/licenses/by/4.0/. The Creative Commons Public Domain Dedication waiver (http://creativecommons.org/publicdomain/zero/1.0/) applies to the data made available in this article, unless otherwise stated in a credit line to the data. 
PACS Picture Archiving and Communication System WBL Weight-bearing line

MA Mechanical axis

ICC Intraclass correlation coefficient

MPTA Medial proximal tibial angle

LDFA Lateral distal femoral angle

JLCA Joint line convergence angle

\section{Background}

Medial opening-wedge high-tibial osteotomy (MOWHTO) is a widely used surgical treatment for medial compartment osteoarthritis (OA) of the knee with a varus deformity $[1,2]$, as well as isolated chondral defects in the medial compartment of the varus knee [3], osteonecrosis in the medial compartment [4], and posttraumatic arthritis and deformity $[5,6]$. It shifts the weight-bearing load from the affected medial compartment to the relatively intact lateral compartment [7]. Proper correction of the deformity is essential to attain satisfactory clinical results after MOWHTO [8]. Under- or overcorrection can lead to inferior clinical outcomes $[9,10]$.

Surgical competence is paramount in MOWHTO to achieve satisfactory outcomes for patients [11]. Although every novice surgeon desires to refine their surgical skills in a short period of time and reduce the learning curve as much as possible in the field of surgery, the accumulation of surgical experience by way of practice is inevitably necessary to reach an adequate level of surgical competence [12]. In MOWHTO, it was reported that a learning curve of approximately 27 cases is necessary to avoid undercorrection [13]. Conversely, in the case of overcorrection, even if 100 cases were completed, adequate prevention was not possible [13]. As such, it can be difficult to attain appropriate alignment even with sufficient surgical experience in MOWHTO [13].

Various preoperative and intraoperative methods have been used to obtain the proper correction for MOWHTO, including the use of a mathematical formula [14], whole lower-extremity radiographs [15], electrocautery cables $[16,17]$, grids [18], fluoroscopy [16, 17], imaging software [19], patient-specific instruments [20], and a navigation system [21]. Despite these efforts, however, some number of outlier cases occur continuously after MOWHTO because alignment corrections made in the supine position cannot exactly represent the postoperative weight-bearing alignment. Recently, the "alignment adjustment under valgus stress technique" protocol to reduce outliers during MOWHTO has been introduced [22]. In this context, after the angle is corrected using the Dugdale method [23], limb alignment is finally adjusted using the intraoperative cable technique by applying valgus stress to the knee joint. Although the reporting authors noted a high accuracy rate [22], questions persist as to whether the manual valgus stress during MWOHTO could show consistency and reproducibility, in particular, for cases treated by a novice surgeon.

In the light of recently gained knowledge, the purpose of this study was to compare the degree of accuracy of surgical correction with use of the "alignment adjustment under valgus stress technique" during MOWHTO between expert and novice surgeons. It was hypothesized that the "alignment adjustment under valgus stress technique" protocol would result in similar acceptable accuracy levels of surgical performance among both expert and novice surgeons during MOWHTO.

\section{Methods}

From June 2018 to November 2019, a total of 84 MOWHTO procedures were performed by either an expert surgeon ( $n=52$ cases, expert group) and a novice surgeon ( $n=32$ cases, novice group) using a contemporary locking plate at a single institution. The expert surgeon had worked as a knee surgeon for 20 years and performed more than 40 MOWHTOs annually for more than 6 years. In contrast, the novice surgeon had completed a basic four-year orthopedic residency and threeyear knee and sports medicine fellowship but lacked experience as an operator of MOWHTO. This study was approved by the institutional review board of the institution. The approved indications for MOWHTO were patients 65 years or younger with isolated medial compartment $\mathrm{OA}$ of the knee and varus malalignment. MOWHTO was contraindicated if a patient had symptomatic OA in the lateral compartment or the patellofemoral joint, inflammatory arthritis, flexion contracture of $15^{\circ}$ or more, knee range of motion less than $120^{\circ}$, joint instability, or a history of knee joint infection [24]. The patients with isolated medial compartment OA with varus deformity under the age of 65 met the inclusion criteria and were included in the study. The patients with traumatic OA, osteonecrosis, follow-up period less than 1 year, and/or incomplete data were excluded. Thus, four cases from the expert group (two knees with a preoperative surgery history on the affected knee and two knees with follow-up loss) and three cases from the novice group (one knee with a previous operation history, one knee with an infection history, and one knee with follow-up loss) were excluded based on the exclusion criteria. Therefore, 48 knees $(n=48$ patients) in the expert group and 29 knees ( $n=29$ patients) in the novice group were enrolled for final analysis.

\section{Surgical technique}

Preoperatively, correction angles were determined in all patients via the Dugdale method [23] using weightbearing full-length hip-ankle radiographs on the Picture 
Archiving and Communication System (PACS) (PI View STAR; Infinitt, Seoul, Korea) [22]. The correction angle was calculated using the angle formed by two lines in the preoperative plan; the first of these ranged from the center of the hip to the so-called Fujisawa point, including $62.5 \%$ of the width of the tibia in the tibia plateau, while the second ranged from the Fujisawa point on the tibial plateau to the center of the ankle joint [25].

A valgus bar was applied to the lateral knee joint preoperatively for the "alignment adjustment under valgus stress technique" (Fig. 1). All surgical procedures were performed under general anesthesia with a tourniquet inflated to $300 \mathrm{mmHg}$. In each case, the pes anserinus

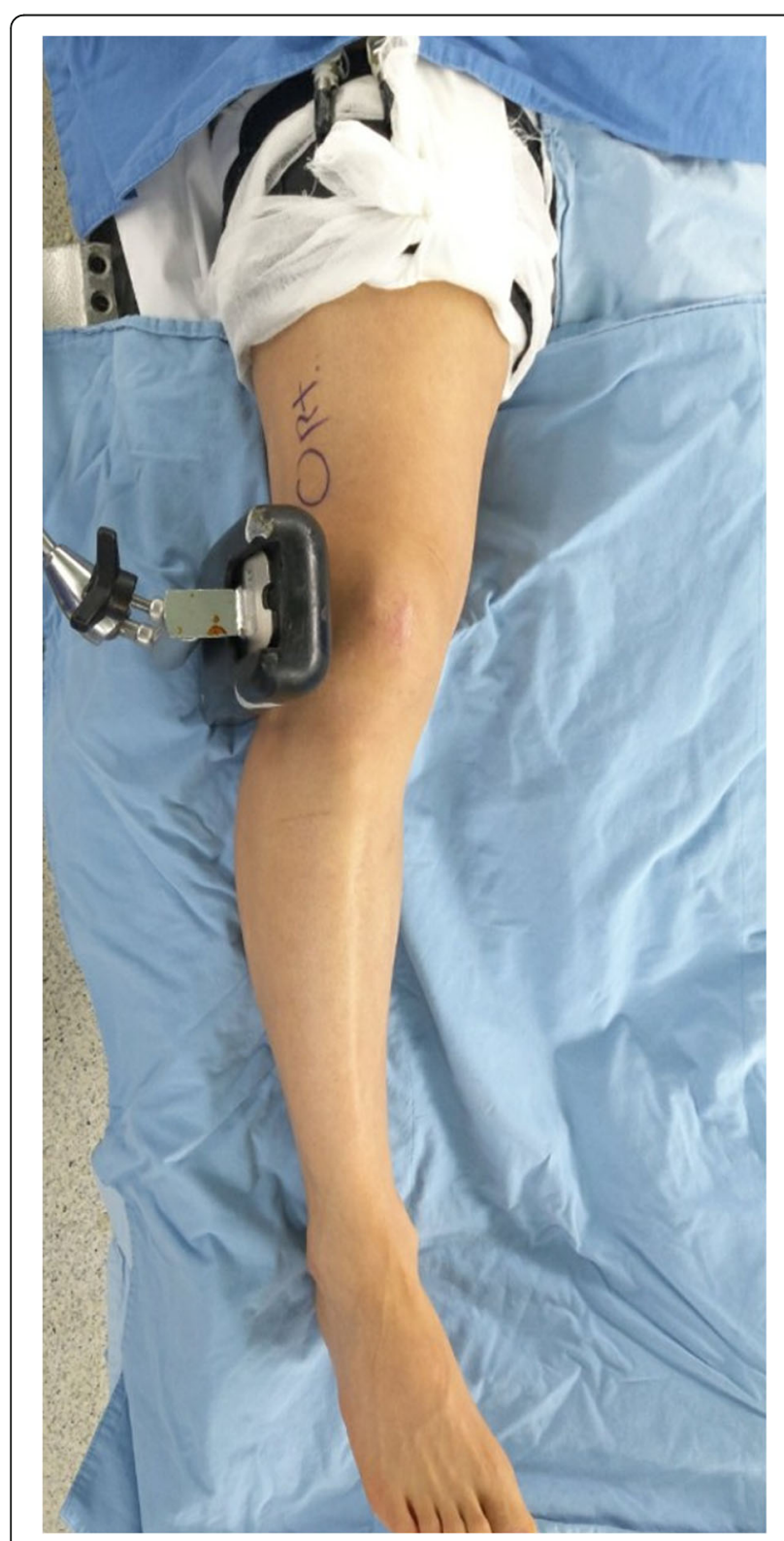

Fig. 1 A valgus bar was applied on the table laterally to the operating knee was identified and released on the medial side of the proximal tibia. Then, the distal portion of the superficial medial collateral ligament was detached from the tibia using a small elevator. Bi-planar MOWHTO was performed according to a technique developed by the Arbeitsgemeinschaft für Osteosynthesefragen knee expert group [26]. A spreader was inserted into the osteotomy site and the osteotomy site was opened gradually to the planned angle. The opening angle was identified from the angle scale on the spreader [26]. The osteotomy gap was left empty without bone graft.

Lower-extremity alignment was assessed by applying valgus stress to the knee joint under an image intensifier following opening of the osteotomy gap as planned by the Dugdale method [22]. Valgus stress was applied to the knee joint with manual maximal force using the valgus bar. By repeating valgus stress several times, the surgeon could feel to what extent valgus stress was appropriate for a specific patient. When valgus stress was applied, the weight-bearing line (WBL) moved to the lateral side (Fig. 2). Then, the alignment was assessed using an electrocautery cable, which passed along the Fujisawa point while applying valgus stress to the knee joint. If the cable passed medial to the Fujisawa point under valgus stress, in a case of undercorrection, the osteotomy gap was opened more to increase the correction angle. On the other hand, in the case of overcorrection, the spreader was closed to reduce the correction angle [22]. Following adjustment under valgus stress, the osteotomy site was fixed with a locking plate (Tomofix; Synthes, Solothurn, Switzerland). (Fig. 3.)

The same postoperative rehabilitation program was applied in both the expert- and novice-performed surgery groups. A quadriceps-setting exercise and continuous passive motion began on the first postoperative day. Partial weight bearing with crutches was allowed from 4 weeks after surgery, and full weight bearing was started 6 weeks after surgery. The protocol was equally applied to all patients including those with underwent meniscal or cartilages procedures.

The radiographic assessment was based on the WBL ratio using weight-bearing full-length hip-ankle radiographs taken preoperatively and at 3 months, 6 months, and 1 year postoperatively. The WBL ratio was defined as the point at which the mechanical axis (MA) passed through the tibial width after drawing a line from the center of the femoral head to the center of the talus dome [15]. The medial and lateral margins of the tibial articular surface were determined as 0 and $100 \%$, respectively. Thus, if the WBL passed through the medial side of the medial edge of the tibia, the value was negative. A PACS was used for radiographic measurements. The acceptable target range of postoperative alignment was a WBL ratio within $62.5 \% \pm 7.5 \%$ from the medial 


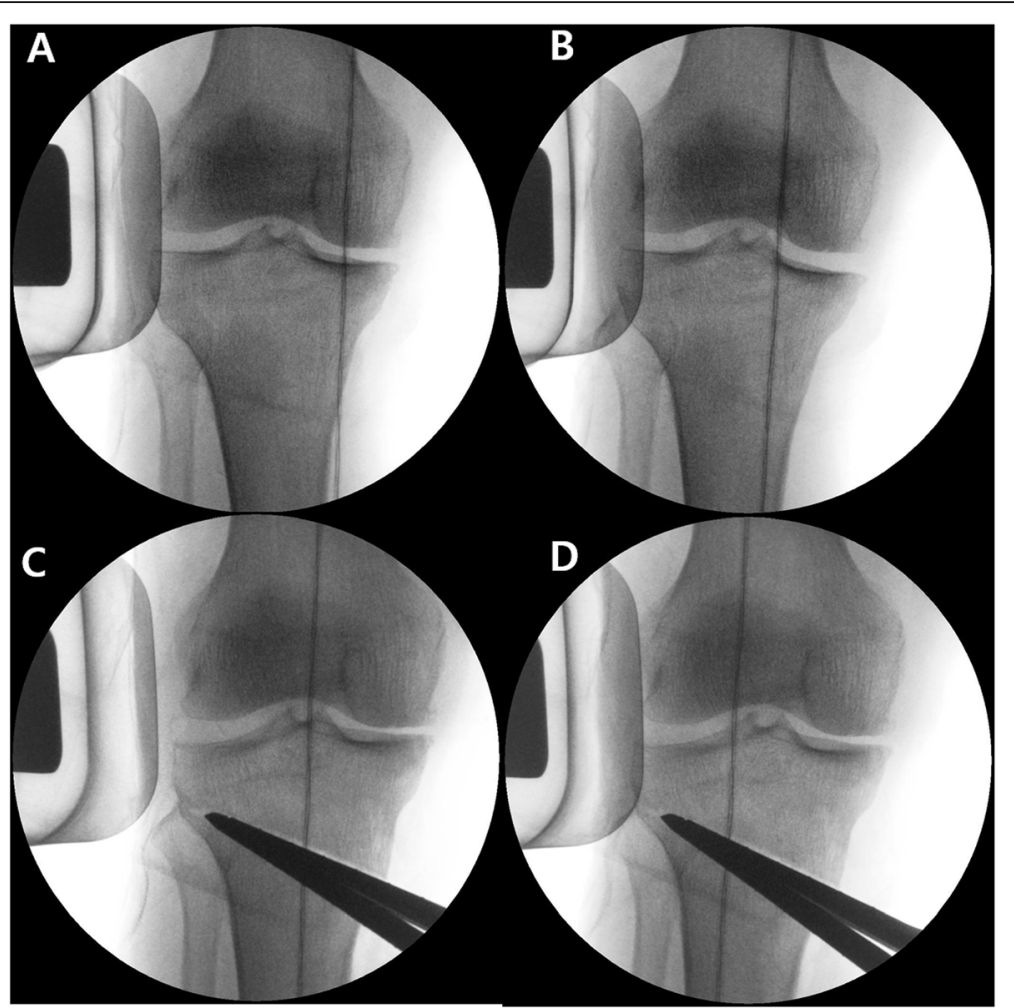

Fig. 2 Change in weight bearing line by applying valgus stress, A Before applying valgus stress in the preosteotomy state, B After applying valgus stress in the preosteotomy state, $\mathbf{C}$ Before applying valgus stress in the postosteotomy state, $\mathbf{D}$ After applying valgus stress in the postosteotomy state

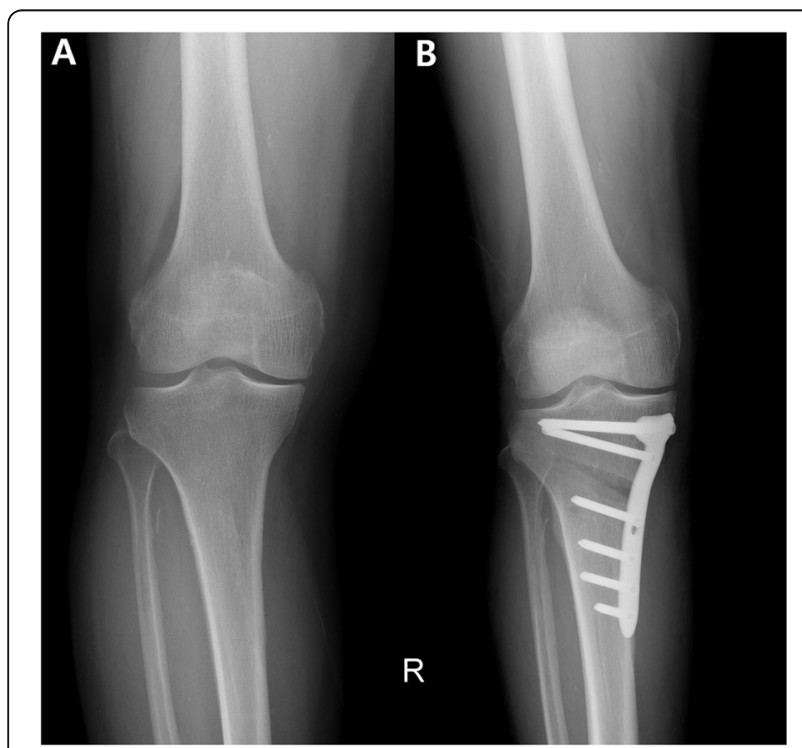

Fig. 3 Preoperative (A) and postoperative (B) radiographs of medial opening wedge high tibial osteotomy edge of the tibia. Meanwhile, an acceptable WBL ratio was defined as a range of 55 to $70 \%$, undercorrection was determined to be a WBL ratio of less than $55 \%$, and overcorrection was defined as that of more than $70 \%$ [27]. The accuracy of surgical performance was compared between the expert- and novice-treated groups using the ratio of normocorrection to outliers. Two orthopedic surgeons who were blinded to the intraoperative procedure and the group measured preoperative and postoperative WBL ratios twice at a two-week interval. Each tester was blinded to the other's measurements and to all patients' data. The average value of the measurements of the two testers was used. Intraobserver and interobserver reliability were calculated for the reliability of the measurement using the intraclass correlation coefficient (ICC). The intraobserver and interobserver ICC values in this study were greater than 0.8 .

The MA, medial proximal tibial angle (MPTA), lateral distal femoral angle (LDFA) and JLCA were also measured preoperatively and at 3 months, 6 months, and 1 year postoperatively. The MPTA was defined as the 


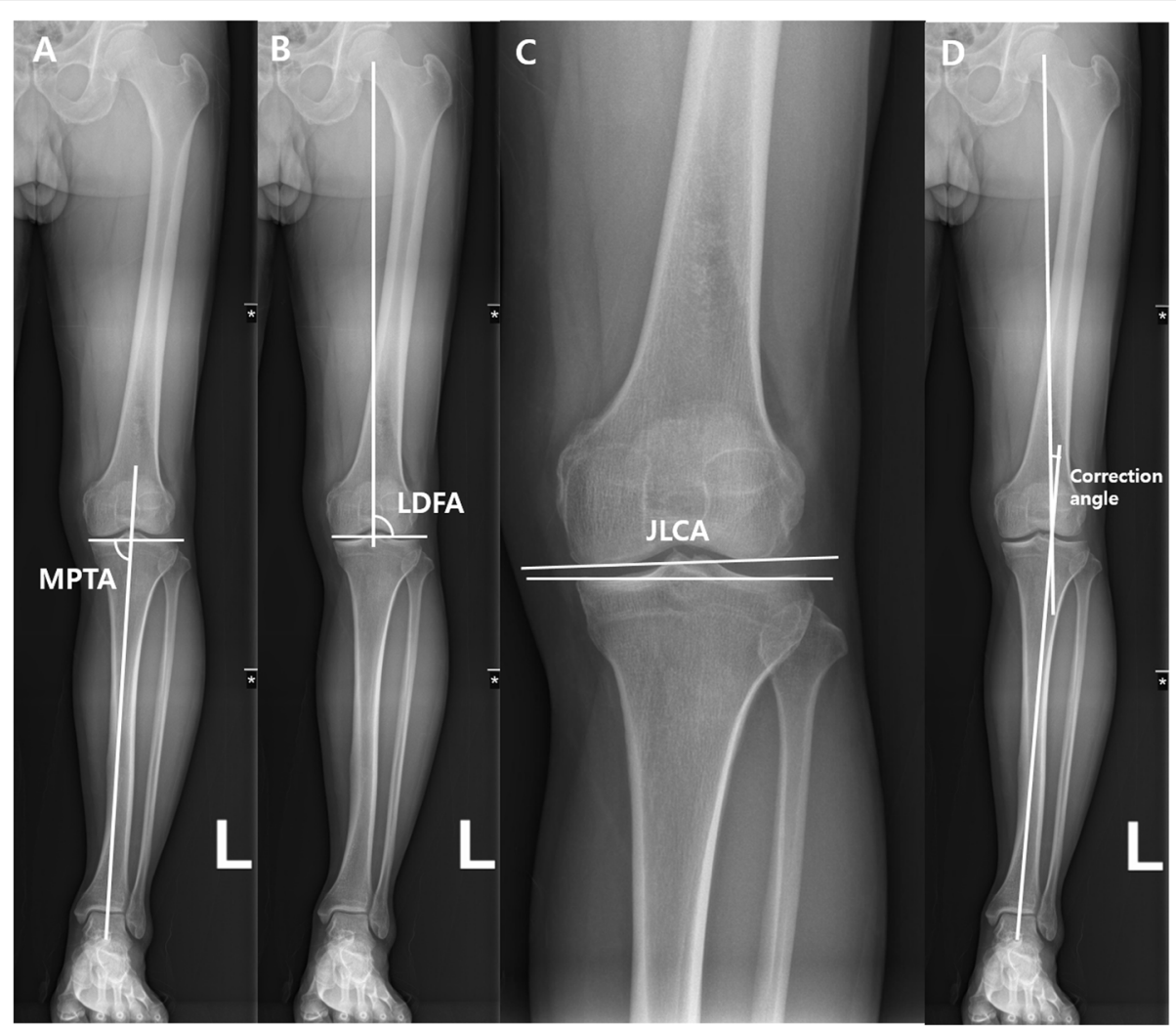

Fig. 4 Radiographic parameters for evaluation of coronal alignment and correction angle of the knee using whole leg anteroposterior radiographs. Medial proximal tibial angle (MPTA) (A), lateral distal femoral angle (LDFA) (B), joint line convergence angle (JLCA) (C), correction angle as planned by Dugdale method (D)

angle that existed between the tibial MA and the articular surface of the proximal tibia. Finally, the LDFA was defined as the angle between the femoral MA and the articular surface of the distal femur [28]. The JLCA was defined as the angle that formed between two articular surfaces of the distal femoral condyle and the tibial plateau [29]. (Fig. 4.)

The knee range of motion (ROM) was measured before and at 1 year after surgery. An orthopedic surgeon who did not participate in the operation measured it using a $60-\mathrm{cm}$ goniometer with the patient in the supine position. Clinical outcomes were evaluated using Western Ontario and McMaster Universities Osteoarthritis Index (WOMAC) scores 1 day before surgery and 1 year after surgery. A WOMAC score with a 15-point improvement based on the minimum clinically important difference (MCID) was assessed at postoperative 1 year [30]. Postoperative complications were also monitored for during the follow-up period.

\section{Statistical analysis}

Demographic data; MA; MPTA; LDFA; JLCA; WBL; and under-, normo-, and overcorrection ratios were compared between the novice and expert groups. The comparison of categorical variables between the two groups was performed using Pearson's chi-squared test. The analysis of continuous normal distribution data was performed using the Student's t-test. The Mann-Whitney $U$ test was used for the analysis of the ordinal categorical and non-normal distribution data. The reproducibility of real weight-bearing alignment of the "alignment adjustment under valgus stress technique" was evaluated by comparing the WBLs recorded between during the intraoperative valgus technique and at 1 year postoperatively. ICC values were used for the intraobserver reliability of the radiographic assessments performed by the two blinded orthopedic surgeons. When referring to the results of a previous study [22] and assuming that a $30 \%$ significant difference in proportion of outliers excluding normocorrection between the two groups. When alpha was set as 0.05 and beta was 0.80 and the ratio of the two groups was 2:1, 22 patients in the novice group and 45 patients in the expert group were required. Therefore, the present study was considered to be appropriately powered to detect a clinically significant difference with greater than $80 \%$ power. The 
Table 1 Comparisons of patient demographics and preoperative deformity data between groups

\begin{tabular}{|c|c|c|c|}
\hline & Expert group $(n=48)$ & Novice group $(n=29)$ & $p$-value \\
\hline \multicolumn{4}{|l|}{ Demographics } \\
\hline Age (years) & $55.8 \pm 7.2$ & $54.8 \pm 6.5$ & 0.528 \\
\hline Sex (\% female) & $42(87.5 \%)$ & $21(72.4 \%)$ & 0.129 \\
\hline $\mathrm{BMI}\left(\mathrm{kg} / \mathrm{m}^{2}\right)$ & $25.9 \pm 3.8$ & $25.1 \pm 3.9$ & 0.344 \\
\hline OA (K-L grade) & & & 0.300 \\
\hline$\leq 2$ & $3(6.3 \%)$ & $5(17.2 \%)$ & \\
\hline 3 & $42(87.5 \%)$ & $22(75.9 \%)$ & \\
\hline 4 & $3(6.3 \%)$ & $2(6.9 \%)$ & \\
\hline \multicolumn{4}{|l|}{ Additional procedures } \\
\hline Partial meniscectomy & $45(93.8 \%)$ & $24(82.8 \%)$ & 0.120 \\
\hline Meniscus repair & $2(4.2 \%)$ & $5(17.2 \%)$ & \\
\hline Microfractures & $44(91.7 \%)$ & $26(89.7 \%)$ & 0.766 \\
\hline Preoperative mechanical axis $\left({ }^{\circ}\right)$ & Varus $6.8 \pm 23.2$ & Varus $6.4 \pm 2.5$ & 0.536 \\
\hline Preoperative WBL ratio (\%) & $16.3 \pm 12.3$ & $21.3 \pm 11.4$ & 0.068 \\
\hline Preoperative JLCA $\left(^{\circ}\right)$ & $3.0 \pm 1.0$ & $2.6 \pm 0.7$ & 0.069 \\
\hline Preoperative MPTA $\left(^{\circ}\right)$ & $83.2 \pm 2.0$ & $83.3 \pm 1.6$ & 0.931 \\
\hline Preoperative LDFA $\left(^{\circ}\right)$ & $87.5 \pm 2.5$ & $88.3 \pm 1.4$ & 0.089 \\
\hline Angle to be corrected by the Dugdale method $\left(^{\circ}\right)$ & $10.2 \pm 2.7$ & $9.7 \pm 2.2$ & 0.337 \\
\hline
\end{tabular}

Statistical Package for the Social Sciences version 21.0 (IBM Corporation, Armonk, NY, USA) was used for statistical analyses. A $p$-value of less than 0.05 was considered to be statistically significant.

\section{Results}

There were no significant differences between the two groups in terms of patient characteristics (Table 1). The preoperative MA was varus $6.8^{\circ}$ in the expert group and varus $6.4^{\circ}$ in the novice group $(p=0.536)$, while the postoperative MA was valgus $2.4^{\circ}$ in the expert group and valgus $2.6^{\circ}$ in the novice group at 1 year after the operation $(p>0.05)$.

The mean preoperative and postoperative one-year WBL ratios were 16.3 and $58.6 \%$, respectively, in the expert group and 21.3 and $60.2 \%$, respectively, in the novice group. No differences in the preoperative and postoperative WBL ratios were observed between the groups (all $p>0.05$ ). JLCA also showed no difference between the two groups from before to 1 year after surgery (all $p>0.05$ ) (Table 2).

The undercorrection rate was $14.6 \%$ in the expert group and $13.8 \%$ in the novice group at 1 year after surgery, while the overcorrection rate was $2.1 \%$ in the expert group and $3.4 \%$ in the novice group. The correction accuracy, presented as the ratio of normocorrection to outliers, was $83.3 \%$ in the expert group and $82.8 \%$ in the novice group. No difference was found during all time points of the follow-up period (all $p>0.05$ ) (Table 3 and Fig. 5). The mean WBL ratio before valgus stress was $58.0 \%(50.0 \sim 68.8 \%)$ in the novice group and $55.5 \%$ $(48.0 \sim 66.0 \%)$ in the expert group $(p>0.05)$. After valgus

Table 2 Comparison of postoperative WBL ratio, HKA angle, and JLCA between the two groups

\begin{tabular}{|c|c|c|c|c|c|c|c|c|c|}
\hline & \multicolumn{3}{|c|}{ Postoperative 3 months } & \multicolumn{3}{|c|}{ Postoperative 6 months } & \multicolumn{3}{|c|}{ Postoperative 1 year } \\
\hline & Expert & Novice & $p$-value & Expert & Novice & $p$-value & Expert & Novice & $p$-value \\
\hline Postoperative WBL ratio (\%) & $58.4 \pm 7.9$ & $61.5 \pm 6.1$ & 0.069 & $58.0 \pm 6.9$ & $60.4 \pm 5.3$ & 0.114 & $57.1 \pm 8.4$ & $60.2 \pm 5.5$ & 0.089 \\
\hline $\begin{array}{l}\text { Postoperative } \\
\text { HKA angle }\end{array}$ & $\begin{array}{l}\text { Valgus } \\
2.5 \pm 1.7\end{array}$ & $\begin{array}{l}\text { Valgus } \\
2.7 \pm 1.5\end{array}$ & 0.608 & $\begin{array}{l}\text { Valgus } \\
2.4 \pm 1.7\end{array}$ & $\begin{array}{l}\text { Valgus } \\
2.7 \pm 1.5\end{array}$ & 0.396 & $\begin{array}{l}\text { Valgus } \\
2.4 \pm 1.9\end{array}$ & $\begin{array}{l}\text { Valgus } \\
2.6 \pm 1.6\end{array}$ & 0.740 \\
\hline Postoperative JLCA $\left(^{\circ}\right)$ & $1.7 \pm 0.9$ & $1.4 \pm 0.7$ & 0.117 & $1.8 \pm 0.8$ & $1.4 \pm 0.8$ & 0.086 & $1.7 \pm 0.9$ & $1.5 \pm 1.0$ & 0.453 \\
\hline
\end{tabular}

WBL Weight-bearing line, HKA Hip-knee-ankle, JLCA Joint line convergence angle.

Values are presented as mean \pm standard deviation. 
Table 3 Comparison of postoperative WBL ratio

\begin{tabular}{|c|c|c|c|}
\hline & Expert $(n=48)$ & Novice $(n=29)$ & $p$-value \\
\hline Postoperative 3 months & & & 0.849 \\
\hline Undercorrection $(<55 \%)$ & $7(14.6 \%)$ & $3(10.3 \%)$ & \\
\hline Normocorrection (55-70\%) & 39 (81.3\%) & $25(86.2 \%)$ & \\
\hline Overcorrection (> 70\%) & $2(4.2 \%)$ & $1(3.4 \%)$ & \\
\hline Postoperative 6 months & & & 0.820 \\
\hline Undercorrection (<55\%) & $9(14.6 \%)$ & $3(10.3 \%)$ & \\
\hline Normocorrection (55-70\%) & 38 (83.3\%) & $25(86.2 \%)$ & \\
\hline Over-correction (> 70\%) & $1(2.1 \%)$ & 1 (3.4\%) & \\
\hline Postoperative 1 year & & & 0.933 \\
\hline Undercorrection $(<55 \%)$ & $7(14.6 \%)$ & $4(13.8 \%)$ & \\
\hline Normocorrection (55-70\%) & $40(83.3 \%)$ & $24(82.8 \%)$ & \\
\hline Overcorrection (> 70\%) & $1(2.1 \%)$ & $1(3.4 \%)$ & \\
\hline$p$-value & 0.971 & 0.994 & \\
\hline
\end{tabular}

stress, the mean WBL ratio was $63.9 \%(55.2 \sim 73.2 \%)$ in the novice group and $62.3 \%(55.0 \sim 70.0 \%)$ in the expert group $(p>0.05)$. After valgus stress, the WBL was migrated laterally by $5.9 \%$ in the novice group and by $6.8 \%$ in the expert group, with no difference between the two groups $(p=0.199)$. The reproducibility of the actual weight-bearing alignment of the technique, presented as the WBL difference between the intraoperative period and 1 year after surgery, shifted to the medial side by $2.1 \%$ in the expert group and $3.4 \%$ in the novice group after surgery $(p=0.460)$. In the novice group, three patients required alignment adjustment after correction. Additional opening of the correction angle was needed in two patients, and further closure of the correction angle was needed in one patient. In the expert group, five patients needed alignment adjustment after correction. An additional increase of correction angle was needed in four patients, and an additional decrease of correction angle was needed in 1 patient.

The preoperative ROM was 135.4 degrees in the novice group and 134.1 degrees in the expert group. One-year postoperative ROM was 136.5 degrees in the novice group and 135.5 degrees in the expert group, and there was no difference between the two groups (all $p>0.05$ ). Preoperatively, there were no differences in the pain, stiffness, or function subscores or the total score of WOMAC (all $p>0.05$, respectively). At 1 year after surgery, all patients in each group showed significant improvements in WOMAC scores following MOWHTO (all $p<0.05$ ), without between-group differences (all $p>0.05$ ) (Table 4). The MCID achievement rate was $83.3 \%$ in the expert group and $79.2 \%$ in the novice group without difference between the two groups $(p>0.05)$.

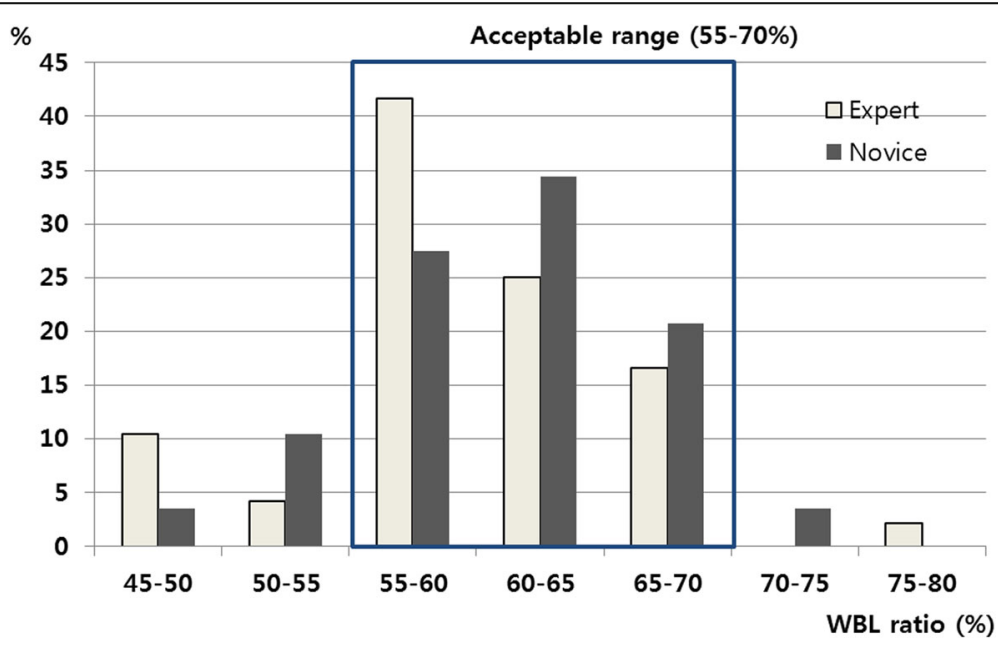

Fig. 5 Distribution of the postoperative WBL ratio between the expert and novice groups 
Table 4 Preoperative and postoperative clinical outcomes

\begin{tabular}{|c|c|c|c|c|c|c|}
\hline & \multicolumn{3}{|c|}{ Preoperative } & \multicolumn{3}{|c|}{ Postoperative 1 year } \\
\hline & Expert & Novice & $p$-value & Expert & Novice & $p$-value \\
\hline WOMAC $^{a}$ & $53.6 \pm 16.1$ & $48.1 \pm 12.0$ & 0.165 & $23.0 \pm 12.0$ & $21.6 \pm 9.8$ & 0.612 \\
\hline Pain & $9.9 \pm 3.6$ & $9.7 \pm 3.2$ & 0.865 & $4.0 \pm 2.6$ & $3.7 \pm 2.5$ & 0.646 \\
\hline Stiffness & $4.7 \pm 2.1$ & $3.7 \pm 2.8$ & 0.079 & $1.6 \pm 1.4$ & $1.5 \pm 1.7$ & 0.730 \\
\hline Function & $39.0 \pm 12.1$ & $34.7 \pm 8.9$ & 0.112 & $17.4 \pm 9.1$ & $16.4 \pm 6.6$ & 0.628 \\
\hline
\end{tabular}

${ }^{\mathrm{a}}$ The WOMAC score

No knee had major complications requiring additional surgery for any reason during the one-year follow-up period. The frequency of lateral hinge fracture was $22.9 \%$ ( $n=11$ cases) in the expert group and $20.7 \%$ ( $n=$ 6 cases) in the novice group $(p=0.819)$. Of the 11 cases in the expert group, 10 cases were type 1 fractures and one case was a type 2 fracture [31]. Meanwhile, all six cases in the novice group were type 1 factures. A fixed rehabilitation protocol that allowed weight bearing at 4 weeks after surgery was applied to patients with lateral hinge fractures. There was no case of correction loss, delayed union, or nonunion in either group.

\section{Discussion}

The most important finding of this study was that a novice surgeon could achieve similar acceptable surgical accuracy to that of an expert surgeon in finding the acceptable correction range by applying "alignment adjustment under valgus stress technique" during MOWHTO.

For a novice surgeon, reducing the learning curve is very important for providing adequate health care to patients $[12,13]$. Since the frequency of MOWHTO is less than that of total knee arthroplasty or anterior cruciate ligament reconstruction, on an occasional basis, more time spent performing MOWHTO procedures may be required to achieve adequate surgical competency [3234]. In MOWHTO, the paramount factors for satisfactory postoperative clinical outcomes are appropriate patient selection [35] and execution of correction [8]. Lee et al. investigated the learning curve of MOWHTO using 100 consecutive cases [13]; to prevent undercorrection, 27 MOWHTO cases were required, while, in the case of overcorrection, it was difficult to prevent such adequately even by 100 cases [13]. It has been reported that joint laxity, especially on the lateral side, could affect lower-extremity alignment following HTO $[2,14]$. The surgical pearls for successful MOWHTO include representation of weight-bearing alignment of the patient, who is in the supine position during surgery [2, $14,36,37]$. In fact, there have been many studies examining the relationship between factors related to joint laxity, including JLCA, to predict the actual standing alignment of MOWHTO patients [2, 14, 36, 37]. However, there continue to be limitations to the prediction of postoperative alignment through radiation measurements related to joint laxity before surgery $[2,14,36$, 37]. Therefore, it is necessary to reproduce the alignment of the standing position while reflecting the joint laxity that differs for each individual in the actual surgical field. In the "alignment adjustment under valgus stress technique" protocol used in this study, the WBL was shifted to the lateral side by applying valgus stress to offset the joint laxity and the amount of adjustment varied from patient to patient. The soft tissue imbalance through valgus-varus stress was imaged before surgery. The degree of soft tissue imbalance through preoperative stress images could be useful to predict joint laxity before surgery [29, 38-40]. The advantage of applying valgus stress intraoperatively is to estimate the weightbearing alignment after correction. By experiencing this technique, we found that the osteotomy site did not open when subjected to valgus stress and only the alignment moved to the lateral side. By using this technique, the novice surgeon could achieve a similar degree of accuracy in surgical performance as that of an expert surgeon. In addition, the degree of reproducibility of the actual standing alignment of the "alignment adjustment under valgus stress technique" also showed no difference between the two groups.

It is difficult to achieve postoperative alignment within the acceptable range following MOWHTO [13, 41-43]. Miniaci et al. [42] stated that when a Fujisawa point of \pm $10 \%$ of WBL ratio after HTO is set as an acceptable range, only $50 \%$ falls into this range. Elsewhere, Marti et al. [41] reported that the accuracy of MOWHTO was also about $50 \%$, with $31 \%$ of cases showing undercorrection and 19\% showing overcorrection. El-Azab et al. [44] reported an undercorrection rate of $8 \%$ and an overcorrection rate of $6 \%$ when a WBL range of 50 to $70 \%$ was set as an acceptable range. In the study of Lee et al. [13], when the acceptable range for WBL was set as 57 to $67 \%$, a total of $61 \%$ of outliers were reported, stratified as $23 \%$ of cases with undercorrection and $38 \%$ of cases with overcorrection. When Stanley et al. [43] set 48 to $68 \%$ as an acceptable range, the conventional technique 
showed $22 \%$ of cases had undercorrection and $15 \%$ had overcorrection. Kim et al. [22] reported that the outlier was about $50 \%$ before using the intraoperative valgus stress technique, yet decreased significantly by about $10 \%$ after the intraoperative valgus technique was adopted. In this study, rates of undercorrection and overcorrection were 13.8 and $3.4 \%$ in the novice group and 14.6 and $2.1 \%$ in the expert group. Both expert and novice surgeons had acceptable levels of accuracy relative to in previous studies [13, 22, 41-43]. In particular, according to the study by Lee et al. [13], it was difficult to prevent overcorrection even if surgical competency with HTO was accumulated. However, in our study, it could be of great significance that the frequency of overcorrection was very low for both novice and expert surgeons when using the intraoperative valgus technique.

Various methods have been reported to obtain an appropriate alignment following HTO, such as full-length radiographs of the lower extremity, imaging software, electrocautery cables, grids, fluoroscopy, and a navigation system [15-17, 19, 21, 45]. However, since the confirmation of alignment during surgery is performed in the supine position, it cannot be recognized without fault as the alignment of the postoperative weightbearing position. Sim et al. [46], as a result of examining patients who underwent MOWHTO, found that there were differences in the MA and WBL on standing and supine radiographs. Specifically, when performing weight-bearing assessments, the WBL ratio moved to $12 \%$ lateral and the FTA angle increased by 1.7 degrees in valgus. Therefore, the authors applied an axial compression force to the heel to reproduce weight-bearing during surgery [46]. Marti et al. [41] also used a push orthoradiography technique with an axial compression force applied to reproduce the weight-bearing status. Kim et al. [22] reported that the weight-bearing status was reproduced through the intraoperative valgus stress technique and that the WBL ratio of $9.6 \%$ moved laterally through the valgus force. In this study, after applying valgus stress, WBL moved laterally by $5.9 \%$ in the novice group and $6.8 \%$ in the expert group, which means that the technique shifted the baseline WBL to a more lateral point, which might have been especially effective in reducing overcorrection outliers. The novice surgeon had outliers (under- and overcorrection) of $17.2 \%$, which were similar to that of $16.7 \%$ among expert surgeons, and showed similar outliers to that of $12 \%$ reported by Kim et al. [22].

The current study had several limitations. First, the proportion of women in both groups was more than $90 \%$. It is a well-known fact that the sex ratio of women in knee osteoarthritis is high in Korea [40, 47, 48]. Second, application of valgus stress using a valgus bar could not reproduce exactly the weight-bearing status, and the degree of valgus stress force could be subjective. However, by measuring valgus stress several times, the surgeon could posit to what extent valgus stress was appropriate for a specific patient. Although both expert and novice surgeons obtained normo-corrections in greater than $80 \%$ of patients with this simple technique, the method is subjective. Third, the follow-up period was as short as 1 year. Comparisons between the two groups during longer-term follow-up can provide more accurate results. Fourth, various confounding factors, such as spatial ability, innate skill, and previous experience, can influence the learning curve in the surgery, but all of these factors were not considered. Therefore, there are limitations in generalizing the results of one novice surgeon to other surgeons. Fifth, although there were no significant differences in the rates of additional procedures such as meniscal or cartilage surgery between the two groups, additional procedures during MOWHTO could affect the clinical outcomes [7, 47, 49, 50]. Finally, a number of variables were measured on radiographs. Therefore, there may be differences attributed to variable radiographic techniques or measurement methods. In this study, to reduce such errors, the full-length radiograph was measured with the patella and both feet in a forward posture and the full extension of the knee joint was applied to reduce the error as much as possible.

Despite these shortcomings, our study revealed that even a novice surgeon in the learning period could obtain an appropriate level of alignment accuracy when implementing MOWHTO through a method that can be easily applied in the operating room. For clinical relevance, as shown by the results of the current study, the use of the "alignment adjustment under valgus stress technique" in the practice of MOWHTO during the learning period of a novice surgeon was reliable and reproducible enough to reduce the learning curve required to perform accurate correction.

\section{Conclusion}

A novice surgeon could have a similar level of surgical accuracy in alignment correction to that of an expert by using the "alignment adjustment under valgus stress technique" protocol during MOWHTO.

\section{Abbreviations}

MOWHTO: Medial opening-wedge high tibial osteotomy; WOMAC: Western Ontario and McMaster Universities Osteoarthritis Index; OA: Osteoarthritis

\section{Acknowledgements}

None.

Authors' contributions

MSK collected the data, performed the measurement and analysis, participated in the study design and drafted the manuscript. IJK, YGS, DCP participated in the study design, supervised the analysis and helped to draft the manuscript. SBH collected the data, performed the measurement. YI 
designed the study, supervised the whole study process and helped to draft and review the manuscript. All authors read and approved the final manuscript.

\section{Funding}

Source of Funding, there was no external funding source in this investigation.

\section{Availability of data and materials}

The datasets used and analyzed during the current study available are from the corresponding author upon reasonable request.

\section{Declarations}

\section{Ethics approval and consent to participate}

All procedures performed in studies involving human participants were in accordance with the ethical standards of the institutional and/or national research committee and with the 1964 Declaration of Helsinki and its later amendments or comparable ethical standards. This study has been approved by the Institutional Review Board (IRB) of Seoul St. Mary's Hospital and informed written consent for participation in the study was obtained.

\section{Consent for publication}

Consent for publication in the study was obtained.

\section{Competing interests}

The authors declare that they have no competing interests.

\section{Author details}

${ }^{1}$ Department of Orthopaedic Surgery, Seoul St. Mary's Hospital, College of Medicine, The Catholic University of Korea, 222, Banpo-daero, Seocho-gu, Seoul 06591, Republic of Korea. '2Department of Orthopaedic Surgery, Eunpyeong St. Mary's Hospital, College of Medicine, The Catholic University of Korea, 1021, Tongil Ro, Eunpyeong-gu, Seoul 03312, Republic of Korea.

Received: 26 March 2021 Accepted: 15 June 2021

Published online: 25 June 2021

\section{References}

1. Hankemeier S, Mommsen P, Krettek C, Jagodzinski M, Brand J, Meyer C, et al. Accuracy of high tibial osteotomy: comparison between open- and closed-wedge technique. Knee Surg Sports Traumatol Arthrosc. 2010;18(10): 1328-33. https://doi.org/10.1007/s00167-009-1020-9.

2. Ogawa H, Matsumoto K, Ogawa T, Takeuchi K, Akiyama H. Preoperative varus laxity correlates with overcorrection in medial opening wedge high tibial osteotomy. Arch Orthop Trauma Surg. 2016;136(10):1337-42. https:// doi.org/10.1007/s00402-016-2521-x.

3. Sabzevari S, Ebrahimpour A, Roudi MK, Kachooei AR. High Tibial osteotomy: a systematic review and current concept. Arch Bone Jt Surg. 2016;4(3):20412.

4. Goshima K, Sawaguchi T, Shigemoto K, Iwai S, Fujita K, Yamamuro Y. Openwedge high tibial osteotomy for spontaneous osteonecrosis of the medial tibial plateau shows excellent clinical outcomes. J Exp Orthop. 2020;7(1):14 https://doi.org/10.1186/s40634-020-00231-z.

5. Biz C, Maso G, Gambato M, Belluzzi E, Pozzuoli A, Favero M, et al. Challenging surgical treatment of displaced articular Tibial plateau fractures: do early knee radiographic features have a predictive value of the mid-term clinical functional outcomes? Orthop Surg. 2019;11(6):1149-62. https://doi. org/10.1111/os.12577.

6. Gao L, Madry H, Chugaev DV, Denti M, Frolov A, Burtsev M, et al. Advances in modern osteotomies around the knee : Report on the Association of Sports Traumatology, Arthroscopy, Orthopaedic surgery, Rehabilitation (ASTAOR) Moscow International Osteotomy Congress 2017. J Exp Orthop. 2019;6:9.

7. Matsunaga D, Akizuki S, Takizawa T, Yamazaki I, Kuraishi J. Repair of articular cartilage and clinical outcome after osteotomy with microfracture or abrasion arthroplasty for medial gonarthrosis. Knee. 2007;14(6):465-71. https://doi.org/10.1016/j.knee.2007.06.008.

8. DeMeo PJ, Johnson EM, Chiang PP, Flamm AM, Miller MC. Midterm followup of opening-wedge high tibial osteotomy. Am J Sports Med. 2010;38(10): 2077-84. https://doi.org/10.1177/0363546510371371.
9. Sharma L, Song J, Felson DT, Cahue S, Shamiyeh E, Dunlop DD. The role of knee alignment in disease progression and functional decline in knee osteoarthritis. Jama. 2001;286(2):188-95. https://doi.org/10.1001/jama.286.2.188.

10. Sprenger TR, Doerzbacher JF. Tibial osteotomy for the treatment of varus gonarthrosis. Survival and failure analysis to twenty-two years. J Bone Joint Surg Am. 2003;85(3):469-74. https://doi.org/10.2106/00004623-20030300000011.

11. Black KP, Armstrong AD, Hutzler L, Egol KA. Quality and safety in Orthopaedics: learning and teaching at the same time: AOA critical issues. J Bone Joint Surg Am. 2015;97(21):1809-15. https://doi.org/10.2106/JBJS.O. 00020.

12. Sadideen $H$, Alvand $A$, Saadeddin $M$, Kneebone R. Surgical experts: born or made? Int J Surg. 2013;11(9):773-8. https://doi.org/10.1016/j.ijsu.2013.07.001.

13. Lee DK, Kim KK, Ham CU, Yun ST, Kim BK, Oh KJ. The learning curve for biplane medial open wedge high Tibial osteotomy in 100 consecutive cases assessed using the cumulative summation method. Knee Surg Relat Res. 2018;30(4):303-10. https://doi.org/10.5792/ksrr.17.064.

14. Lee DK, Wang JH, Won Y, Min YK, Jaiswal S, Lee BH, et al. Preoperative latent medial laxity and correction angle are crucial factors for overcorrection in medial open-wedge high tibial osteotomy. Knee Surg Sports Traumatol Arthrosc. 2020;28(5):1411-8. https://doi.org/10.1007/s001 67-019-05502-6.

15. Lee DH, Han SB, Oh KJ, Lee JS, Kwon JH, Kim Jl, et al. The weight-bearing scanogram technique provides better coronal limb alignment than the navigation technique in open high tibial osteotomy. Knee. 2014;21(2):451-5. https://doi.org/10.1016/j.knee.2012.09.003.

16. Hsu RW, Himeno S, Coventry MB, Chao EY. Normal axial alignment of the lower extremity and load-bearing distribution at the knee. Clin Orthop Relat Res. 1990;1:215-27.

17. Liodakis E, Kenawey M, Liodaki E, Mommsen P, Krettek C, Hankemeier S. The axis-board: an alternative to the cable technique for intraoperative assessment of lower limb alignment. Technol Health Care. 2010;18(3):16571. https://doi.org/10.3233/THC-2010-0579.

18. Lützner J, Gross AF, Günther KP, Kirschner S. Precision of navigated and conventional open-wedge high tibial osteotomy in a cadaver study. Eur J Med Res. 2010;15(3):117-20. https://doi.org/10.1186/2047-783X-15-3-117.

19. Lee YS, Kim MK, Byun HW, Kim SB, Kim JG. Reliability of the imaging software in the preoperative planning of the open-wedge high tibial osteotomy. Knee Surg Sports Traumatol Arthrosc. 2015;23(3):846-51. https:// doi.org/10.1007/s00167-013-2700-z.

20. Tardy N, Steltzlen C, Bouguennec N, Cartier JL, Mertl P, Batailler C, et al. Is patient-specific instrumentation more precise than conventional techniques and navigation in achieving planned correction in high tibial osteotomy? Orthop Traumatol Surg Res. 2020;106(8):S231-s6. https://doi.org/10.1016/j. otsr.2020.08.009.

21. Kendoff D, Board TN, Citak M, Gardner MJ, Hankemeier S, Ostermeier S, et al. Navigated lower limb axis measurements: influence of mechanical weight-bearing simulation. J Orthop Res. 2008;26(4):553-61. https://doi. org/10.1002/jor.20510.

22. Kim MS, Son JM, Koh IJ, Bahk JH, In Y. Intraoperative adjustment of alignment under valgus stress reduces outliers in patients undergoing medial opening-wedge high tibial osteotomy. Arch Orthop Trauma Surg. 2017;137(8):1035-45. https://doi.org/10.1007/s00402-017-2729-4.

23. Dugdale TW, Noyes FR, Styer D. Preoperative planning for high tibial osteotomy. The effect of lateral tibiofemoral separation and tibiofemoral length. Clin Orthop Relat Res. 1992;1:248-64.

24. Lee DC, Byun SJ. High tibial osteotomy. Knee Surg Relat Res. 2012;24(2):619. https://doi.org/10.5792/ksrr.2012.24.2.61.

25. Kubota M, Ohno R, Sato T, Yamaguchi J, Kaneko H, Kaneko K, et al. The medial proximal tibial angle accurately corrects the limb alignment in openwedge high tibial osteotomy. Knee Surg Sports Traumatol Arthrosc. 2019; 27(8):2410-6. https://doi.org/10.1007/s00167-018-5216-8.

26. Lobenhoffer P, Agneskirchner JD. Improvements in surgical technique of valgus high tibial osteotomy. Knee Surg Sports Traumatol Arthrosc. 2003; 11(3):132-8. https://doi.org/10.1007/s00167-002-0334-7.

27. Na YG, Eom SH, Kim SJ, Chang MJ, Kim TK. The use of navigation in medial opening wedge high tibial osteotomy can improve tibial slope maintenance and reduce radiation exposure. Int Orthop. 2016;40(3):499-507. https://doi.org/10.1007/s00264-015-2880-X.

28. Song KY, Koh IJ, Kim MS, Choi NY, Jeong JH, In Y. Early experience of lateral hinge fracture during medial opening-wedge high tibial osteotomy: 
incidence and clinical outcomes. Arch Orthop Trauma Surg. 2020;140(2): 161-9. https://doi.org/10.1007/s00402-019-03237-0.

29. Lee OS, Lee SH, Lee YS. Does coronal knee and ankle alignment affect recurrence of the Varus deformity after high Tibial osteotomy? Knee Surg Relat Res. 2018;30(4):311-8. https://doi.org/10.5792/ksrr.18.035.

30. Dowsey MM, Choong PF. The utility of outcome measures in total knee replacement surgery. Int J Rheumatol. 2013;2013:506518.

31. Takeuchi R, Ishikawa H, Kumagai K, Yamaguchi Y, Chiba N, Akamatsu Y, et al. Fractures around the lateral cortical hinge after a medial opening-wedge high tibial osteotomy: a new classification of lateral hinge fracture. Arthroscopy. 2012;28(1):85-94. https://doi.org/10.1016/j.arthro.2011.06.034.

32. Herzog MM, Marshall SW, Lund JL, Pate V, Mack CD, Spang JT. Trends in incidence of $\mathrm{ACL}$ reconstruction and concomitant procedures among commercially insured individuals in the United States, 2002-2014. Sports Health. 2018;10(6):523-31. https://doi.org/10.1177/1941738118803616.

33. Koh IJ, Kim MW, Kim JH, Han SY, In Y. Trends in high Tibial osteotomy and knee arthroplasty utilizations and demographics in Korea from 2009 to 2013. J Arthroplast. 2015;30(6):939-44. https://doi.org/10.1016/j.arth.2015.01. 002.

34. Niinimäki TT, Eskelinen $A$, Ohtonen $P$, Junnila $M$, Leppilahti J. Incidence of osteotomies around the knee for the treatment of knee osteoarthritis: a 22year population-based study. Int Orthop. 2012;36(7):1399-402. https://doi. org/10.1007/s00264-012-1508-7.

35. Sohn S, Koh IJ, Kim MS, Kang BM, In Y. What factors predict patient dissatisfaction after contemporary medial opening-wedge high Tibial osteotomy? J Arthroplast. 2020;35(2):318-24. https://doi.org/10.1016/j.arth.2 019.09.026.

36. Kim JE, Kim DH, Lee Jl, Choi HG, Jung YS, Lee SH, et al. Difference of preoperative varus-valgus stress radiograph is effective for the correction accuracy in the preoperative planning during open-wedge high tibial osteotomy. Knee Surg Sports Traumatol Arthrosc. 2020;29:1035.

37. Park JG, Kim JM, Lee BS, Lee SM, Kwon OJ, Bin SI. Increased preoperative medial and lateral laxity is a predictor of overcorrection in open wedge high tibial osteotomy. Knee Surg Sports Traumatol Arthrosc. 2020;28(10): 3164-72. https://doi.org/10.1007/s00167-019-05805-8.

38. Kim JE, Kim DH, Lee Jl, Choi HG, Jung YS, Lee SH, et al. Difference of preoperative varus-valgus stress radiograph is effective for the correction accuracy in the preoperative planning during open-wedge high tibial osteotomy. Knee Surg Sports Traumatol Arthrosc. 2021;29(4):1035-44. https://doi.org/10.1007/s00167-020-06076-4.

39. Lee OS, Elazab A, Lee YS. Preoperative Varus-Valgus stress angle difference is valuable for predicting the extent of medial release in Varus deformity during Total knee arthroplasty. Knee Surg Relat Res. 2019;31(1):12-8. https:// doi.org/10.5792/ksrr.18.033

40. Lee OS, Lee ES, Lee YS. Disparity between preoperative target correction amount and postoperative correction amount in open wedge high Tibial osteotomy. Knee Surg Relat Res. 2019;31(2):126-31. https://doi.org/10.5792/ ksrr.18.034.

41. Marti CB, Gautier E, Wachtl SW, Jakob RP. Accuracy of frontal and sagittal plane correction in open-wedge high tibial osteotomy. Arthroscopy. 2004; 20(4):366-72. https://doi.org/10.1016/j.arthro.2004.01.024.

42. Miniaci A, Ballmer FT, Ballmer PM, Jakob RP. Proximal tibial osteotomy. A new fixation device. Clin Orthop Relat Res. 1989;1:250-9.

43. Stanley JC, Robinson KG, Devitt BM, Richmond AK, Webster KE, Whitehead TS, et al. Computer assisted alignment of opening wedge high tibial osteotomy provides limited improvement of radiographic outcomes compared to flouroscopic alignment. Knee. 2016;23(2):289-94. https://doi. org/10.1016/j.knee.2015.12.006

44. El-Azab HM, Morgenstern M, Ahrens P, Schuster T, Imhoff AB, Lorenz SG. Limb alignment after open-wedge high tibial osteotomy and its effect on the clinical outcome. Orthopedics. 2011;34(10):e622-8. https://doi.org/10.392 8/01477447-20110826-02.

45. Hawi N, Liodakis E, Suero EM, Meller R, Citak M, Krettek C. A cadaver study comparing intraoperative methods to analyze lower limb alignment. Skelet Radiol. 2014;43(11):1577-81. https://doi.org/10.1007/s00256-014-1972-9.

46. Sim JA, Kwak JH, Yang SH, Choi ES, Lee BK. Effect of weight-bearing on the alignment after open wedge high tibial osteotomy. Knee Surg Sports Traumatol Arthrosc. 2010;18(7):874-8. https://doi.org/10.1007/s00167-009-1 000-0.

47. Lee HI, Park D, Cho J. Clinical and radiological results with second-look arthroscopic findings after open wedge high Tibial osteotomy without arthroscopic procedures for medial meniscal root tears. Knee Surg Relat Res. 2018;30(1):34-41. https://doi.org/10.5792/ksrr.17.035.

48. Yoo JH, Oh HC, Park SH, Kim JK, Kim SH. Does obesity affect clinical and radiological outcomes in minimally invasive Total knee arthroplasty? Minimum 5-year follow-up of minimally invasive TKA in obese patients. Clin Orthop Surg. 2018;10(3):315-21. https://doi.org/10.4055/cios.2018.10.3.315.

49. Jing L, Liu K, Wang X, Wang X, Li Z, Zhang X, et al. Second-look arthroscopic findings after medial open-wedge high tibial osteotomy combined with all-inside repair of medial meniscus posterior root tears. J Orthop Surg (Hong Kong). 2020;28:2309499019888836.

50. Kim JK, Vaidya R, Lee SK, Yu J, Park JY, Ro DH, et al. Clinical and radiological changes after microfracture of knee chondral lesions in middle-aged Asian patients. Clin Orthop Surg. 2019;1 1(3):282-90. https://doi.org/10.4055/cios.2 019.11.3.282.

\section{Publisher's Note}

Springer Nature remains neutral with regard to jurisdictional claims in published maps and institutional affiliations.
Ready to submit your research? Choose BMC and benefit from:

- fast, convenient online submission

- thorough peer review by experienced researchers in your field

- rapid publication on acceptance

- support for research data, including large and complex data types

- gold Open Access which fosters wider collaboration and increased citations

- maximum visibility for your research: over $100 \mathrm{M}$ website views per year

At BMC, research is always in progress.

Learn more biomedcentral.com/submissions 